\section{Pannus subvalvular como causa de desproporción tardía prótesis-paciente después de sustitución de la válvula aórtica}

\section{Subvalvular pannus as cause of late mismatch after aortic valve replacement}

\section{Sr. Editor:}

La desproporción entre el tamaño de la prótesis utilizada para sustituir una válvula cardiaca y el tamaño del paciente se conoce como «mismatch» de la prótesis en la literatura anglosajona. El significado clínico de esta circunstancia, responsabilizada por algunos como causa de obstrucción al vaciado ventricular, ha sido motivo de debate a lo largo de los últimos años, relacionándose con una mayor morbilidad postoperatoria y peor supervivencia ${ }^{1}$. El mecanismo causal comúnmente evocado es el insuficiente orificio valvular efectivo de la prótesis como consecuencia de su pequeño tamaño o de particularidades de su diseño ${ }^{1-3}$. Sin embargo, otra posible causa de desproporción (mismatch) prótesis-paciente, no claramente descrita ni demostrada gráficamente, es la formación de pannus después de implantar la prótesis ${ }^{2,3}$. A continuación, exponemos el caso de una paciente diagnosticada de desproporción (mismatch) de prótesis valvular aórtica que en la intervención se comprobó era secundario al crecimiento excesivo de pannus perivalvular, y mostramos lo que este fenómeno supone bajo un punto de vista gráfico.

Mujer de 74 años, con antecedentes de asma bronquial y amigdalitis de repetición en la infancia, diagnosticada de valvulopatía mitral reumática, con estenosis grave, y llevada a comisurotomía abierta hace 30 años. Diez años más tarde fue reintervenida por estenosis aórtica grave, realizándose sustitución valvular aórtica por prótesis mecánica Carbomedics n. ${ }^{\circ} 19$. Desde entonces siguió revisiones periódicas, encontrándose asintomática hasta hace 2 años, cuando comienza con disnea de esfuerzo progresiva hasta alcanzar un grado III/Iv de la NYHA, requiriendo varios ingresos por insuficiencia cardiaca en el último año. En ecocardiogramas sucesivos, se evidenció progresión de la valvulopatía mitral hasta presentar estenosis e insuficiencia graves, así como insuficiencia tricúspide e hipertensión pulmonar graves. Además, se detectaron signos ecocardiográficos de estenosis de la prótesis aórtica (velocidad máxima: $5 \mathrm{~m} / \mathrm{s}$, gradiente máximo: $68 \mathrm{mmHg}$, y medio: $36 \mathrm{mmHg}$ ) a pesar de un movimiento normal de los hemidiscos, lo que sugería la presencia de desproporción aórtica. Dada la sintomatología de la paciente y las lesiones valvulares, se indicó la cirugía para corregir la enfermedad mitrotricúspide y explorar la prótesis aórtica. El registro intraoperatorio simultáneo de las presiones del ventrículo izquierdo y aorta confirmó la existencia de un gradiente pico mayor de $60 \mathrm{mmHg}$. Se realizó sustitución valvular mitral, anuloplastia tricúspide y se exploró la prótesis aórtica, evidenciando un ancho rodete subvalvular de pannus que reducía de manera importante el orificio valvular aórtico efectivo y que solo fue apreciable en toda su extensión tras explantar la prótesis aórtica (fig. 1). Se resecó el rodete subvalvular y se implantó una nueva
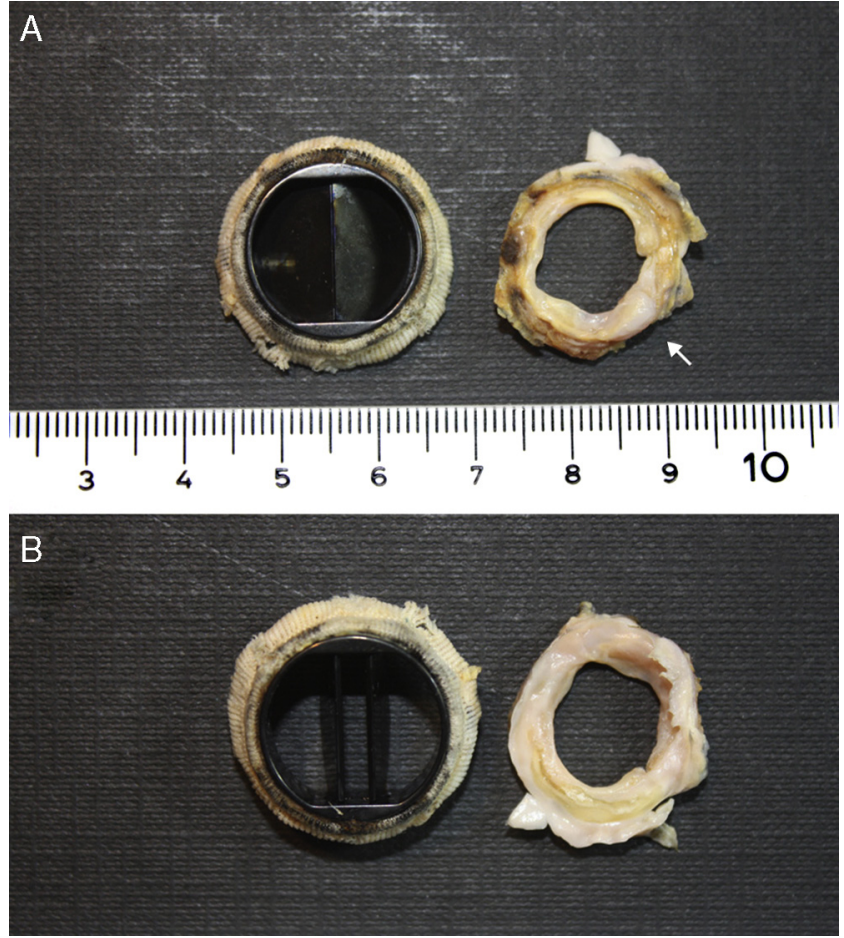

Figura 1 Imagen de la prótesis aórtica desde ambas facetas: arterial (A) y ventricular (B). El tejido de pannus (flecha) se extiende alrededor de la circunferencia, siendo visible la considerable reducción del diámetro en el orificio valvular efectivo de la prótesis valvular aórtica.

prótesis mecánica (On-X n. ${ }^{\circ}$ 19, On-X Life Technologies Inc, Austin, Texas) registrando un gradiente transprotésico pico de $11 \mathrm{mmHg}$ después de la salida de circulación extracorpórea. El postoperatorio transcurrió sin incidencias y la paciente fue egresada en 7 días.

La desproporción entre el tamaño de la prótesis valvular implantada y la superficie corporal del paciente en el que se implanta se denomina desproporción protésica. La existencia de esta desproporción prótesis-paciente a nivel valvular aórtico es una entidad a considerar en aquellos pacientes intervenidos de sustitución valvular en los que se detecta un gradiente transprotésico anormalmente elevado ${ }^{2-4}$. En sentido estricto, esta desproporción (mismatch) define la insuficiencia del orificio valvular efectivo para adaptar el gasto cardiaco necesario para un determinado paciente, especialmente en situaciones de estrés hemodinámico, y excluye la participación de cualquier mecanismo extrínseco. Es una circunstancia especialmente frecuente en pacientes portadores de prótesis de pequeño diámetro o de gran superficie corporal ${ }^{1-4}$. No obstante, también debe contemplarse la posibilidad de que la desproporción se deba a un exceso de pannus, especialmente en prótesis con parámetros ecocardiográficos inicialmente normales ${ }^{2,4}$, como en el caso que aquí se presenta. El pannus es un tejido fibroso compuesto por fibras de colágeno y fibroblastos que se forma después de implantar cualquier prótesis en las zonas de estasis hemática ${ }^{1,2,5}$. La proliferación del pannus está influida por la posición de la prótesis, el diseño y el tamaño, el comportamiento hidrodinámico, la biocompatibilidad de los 
materiales, la técnica quirúrgica, el grado de anticoagulación y la intensidad de la reacción inflamatoria generada después del implante valvular. En la mayoría de los casos, este tejido se limita a la zona del anillo valvular, donde asienta la prótesis ${ }^{1,5,6}$. Sin embargo, puede desarrollarse en exceso, tanto a nivel supra. como subvalvular, siendo causa de disfunción extrínseca de la prótesis en forma de estenosis, bloqueo de velos o hemidiscos, o incluso convertirse en foco embolígeno simulando una trombosis valvular o una endocarditis ${ }^{5}$.

Por medio de este caso clínico creemos relevante mostrar cómo la desproporción o mismatch protésico no es solo un fenómeno inherente al tamaño de la prótesis, sino que también puede deberse al crecimiento excesivo de pannus, fenómeno frecuente y con las mismas potenciales consecuencias en forma de un gradiente transprotésico elevado. Las mejorías en el diseño de las prótesis que, como la On-X, minimizan la formación de pannus y preservan el orificio valvular suponen un avance significativo en su prevención ${ }^{7-9}$.

\section{Bibliografía}

1. Dumesnil JG, Pibarot P. Prosthesis-patient mismatch: An update. Curr Cardiol Rep. 2011;13:250-7.

2. Valencia Sánchez JS, Arriaga Nava R, Martínez Enriquez A, et al. Medical-surgical management of left heart valve prosthesis dysfunction due to obstruction. Eleven year's experience. Arch Inst Cardiol Mex. 1999;69:127-33.

3. Kaminishi Y, Misawa Y, Kobayashi J, et al. Patient-prosthesis mismatch in patients with aortic valve replacement. Gen Thorac Cardiovasc Surg. 2013;61:274-9.

4. Espinola Zavaleta N, de la Rosa G, Tamayo Rebolledo E, et al. Valve prostheses and transesophageal echocardiography. Arch Inst Cardiol Mex. 1995;65:49-56.
5. Tabet JY, Bouvier E, Cormier B, et al. Unusual cause of stroke after mitral valve replacement. Circulation. 2010;122: e484-5.

6. Teshima H, Hayashida N, Yano H, et al. Obstruction of St. Jude Medical valves in the aortic position: histology and immunohistochemistry of pannus. J Thorac Cardiovasc Surg. 2003;126: 401-7.

7. Wippermann J, Albes JM, Madershahian N, et al. Three years' experience with the on- $\mathrm{X}$ conform- $\mathrm{X}$ bileaflet prosthesis for 'atrialized' mitral valve replacement: A preliminary report. J Heart Valve Dis. 2005;14:637-43.

8. Ely JL, Emken MR, Accuntius JA, et al. Pure pyrolytic carbon: Preparation and properties of a new material, On-X carbon for mechanical heart valve prostheses. J Heart Valve Dis. 1998; 7:626-32.

9. Chambers JC, Ely JL. Early and late echocardiographic performance of the On-X AorticValve, presented at Advances in Cardiac Surgery 2001 in San Diego, CA, May 5, 20.

María Elena Arnáiz-García ${ }^{a, *}$, Jose María. González-Santos ${ }^{a}$, Adolfo Arévalo-Abascal ${ }^{\mathrm{a}}$, María Bueno-Codoñer ${ }^{\mathrm{a}}$, María José Dalmau-Sorlía, Javier López-Rodríquez ${ }^{\mathrm{a}}$ y Antonio Arribas-Jiménez ${ }^{\mathrm{b}}$

a Servicio de Cirugía Cardiaca, Hospital Clínico

Universitario de Salamanca, Salamanca, España

b Servicio de Cardiología, Hospital Clínico Universitario de Salamanca, Salamanca, España

* Autora para correspondencia. Servicio de Cirugía Cardiaca, Hospital Clínico Universitario de Salamanca, Paseo San Vicente 58-182.37007 Salamanca, España.

Teléfono: +923 291263; fax: +923 291263.

Correo electrónico: elearnaiz@hotmail.com (M.E. Arnáiz-García).

http://dx.doi.org/10.1016/j.acmx.2014.09.006 